\title{
On the Number of Criteria Needed to Decide Pareto Optimality ${ }^{1}$
}

\author{
Matthias EhrgotT ${ }^{2}$ \\ STEFAN NICKEL ${ }^{3}$
}

\begin{abstract}
In this paper we address the question of how many objective functions are needed to decide whether a given point is a Pareto optimal solution for a multicriteria optimization problem. We extend earlier results showing that the set of weakly Pareto optimal points is the union of Pareto optimal sets of subproblems and show their limitations. We prove that for strictly quasi-convex problems in two variables Pareto optimality can be decided by consideration of at most three objectives at a time. Our results are based on a geometric characterization of Pareto, strict Pareto and weak Pareto solutions and Helly's Theorem. We also show that a generalization to quasi-convex objectives is not possible, and state a weaker result for this case. Furthermore, we show that a generalization to strictly Pareto optimal solutions is impossible, even in the convex case.
\end{abstract}

Keywords. Multicriteria optimization, Pareto optimality, strictly quasi-convex functions, number of objectives.

\footnotetext{
${ }^{1}$ We thank Ansgar Weißler for helpful comments and his contribution to the examples. We also thank two anonymous referees for their pertinent comments on a previous version of this paper.

${ }^{2}$ Assistant Professor, Fachbereich Mathematik Universität Kaiserslautern, Postfach 3049, 67663 Kaiserslautern, Germany, e-mail: ehrgott@mathematik.uni-kl.de, fax: (49) 63129082

${ }^{3}$ Assistant Professor, Fachbereich Mathematik Universität Kaiserslautern, Postfach 3049, 67663 Kaiserslautern, Germany, e-mail: nickel@mathematik.uni-kl.de, fax: (49) 63129082
} 


\section{Introduction}

In multiple criteria optimization, optimal decisions have to be found in the presence of several conflicting criteria. A decision is only considered optimal if an improvement of one criterion implies a deterioration of at least one other criterion. The corresponding outcomes are called efficient points, the solutions Pareto optimal solutions.

One topic in the investigation of multiple criteria optimization problems is the determination of those objectives that determine the set of Pareto optimal solutions. Several authors have worked in that field: Gal and Leberling in [1],[2] and [3] introduced the notion of nonessential objectives and presented methods for their determination in the case of linear multicriteria problems. Nonessential objectives can be dropped without changing the set of Pareto optimal solutions. Gal and Hanne in [4] investigated the consequences of dropping nonessential objectives in the search for a final compromise solution by MCDM methods. A more general concept of interdependent criteria has been discussed by Carlsson and Fuller in [5], see also [6].

Apart from research about nonessential or interdependent objectives, some authors were interested in the structure of the set of weakly Pareto optimal points. The results obtained by Ward in [7] and by Malivert and Boissard in [8] show that in problems with $n$ variables considering subproblems with at most $n+1$ criteria is sufficient to determine the whole set of weakly Pareto optimal points of a multicriteria optimization problem with convex criteria. This theory is more general than interdependent objectives because the results also hold in the absence of nonessential criteria, as will be demonstrated by an example presented in this paper.

We continue this research in the sense that we determine the number of objectives which are necessary to prove Pareto (not only weak Pareto) optimality for a given point. The result that for problems with 2 variables in fact only (at most) 3 criteria have to be considered simultaneously leads to considerable advances for multiple criteria problems where the 
number of objectives $Q$ is much larger than the dimension of the decision space (2). This situation arises in location theory, see [9], [10], and [7]. Usually not one single person decides about the location of a new facility but a group of $Q$ decision makers. Each of the $Q$ decision makers gives his personal view of the location problem by means of a specific objective function. Typical objective functions in location theory are the weighted sum or the weighted maximum of the distances of existing facilities to the new one. Each decision maker may choose his individual set of weights as well as the type of the objective function. Therefore, in location theory we deal with a set of convex objective functions on the plane.

The paper is organized as follows. In Section 2 the multiple criteria optimization problem (MOP) is introduced. We present the definitions of (strict, weak) Pareto optimality and give a geometric characterization of all three optimality concepts based on level sets and level curves. In Section 3 we show, by means of an example, that results obtained in [7] and [8] for convex and strictly quasi-convex functions cannot be generalized to quasi-convex functions. We prove a weaker result in this case. The main part is Section 4, where we consider the strictly quasi-convex case. The main theorem (Theorem 4.3) extends a result of [8] for Pareto optimal solutions. The proof of this main result provides a prototype polynomial time algorithm to check Pareto optimality for strictly quasi-convex (MOP), which has the same complexity as the determination of the weakly Pareto optimal set in [8]. Finally, an illustrative example is given in Section 5 and conclusions are stated in Section 6.

\section{Pareto Optimality}

In this section we consider the general multiple criteria optimization problem

$$
\min _{x \in \mathcal{X}} f(x)
$$


where $\mathcal{X} \subseteq \mathbb{R}^{n}$ is the feasible set and $f=\left(f^{1}, \ldots, f^{Q}\right): \mathbb{R}^{n} \rightarrow \mathbb{R}^{Q}$ is the criterion mapping. The component functions $f^{1}, \ldots, f^{Q}$ are the criteria or objective functions. To avoid technicalities we assume throughout that all functions are defined on the whole space $\mathbb{R}^{n}$. The index set of criteria will be denoted by $\mathcal{Q}:=\{1, \ldots, Q\}$.

Optimizing the $Q$ objective functions means minimization in $\mathbb{R}^{Q}$. Therefore, instead of the canonical order in $\mathbb{R}$, we consider three types of partial orders in $\mathbb{R}^{Q}$. Accordingly, we have three different types of optimality.

A point $x \in \mathcal{X}$ is called

- strict Pareto solution (of (MOP)) or strictly Pareto optimal if there is no $\bar{x} \in \mathcal{X} \backslash\{x\}$ satisfying

$$
f(\bar{x}) \leq f(x) \text {, i.e. } f^{i}(\bar{x}) \leq f^{i}(x) \forall i=1, \ldots, Q
$$

- Pareto solution (of (MOP)) or Pareto optimal if there is no $\bar{x} \in \mathcal{X}$ dominating $x$, i.e. satisfying

$$
f(\bar{x}) \leq f(x), \text { and } f(\bar{x}) \neq f(x)
$$

- weak Pareto solution (of (MOP)) or weakly Pareto optimal if there is no $\bar{x} \in \mathcal{X}$ satisfying

$$
f(\bar{x})<f(x) \text {, i.e. } f^{i}(\bar{x})<f^{i}(x) \forall i=1, \ldots, Q \text {. }
$$

The sets of all strict Pareto, Pareto and weak Pareto solutions are denoted by $\mathcal{X}_{s-P a r}$, $\mathcal{X}_{P a r}$, and $\mathcal{X}_{w-P a r}$, respectively. For $\left\{q_{1}, \ldots, q_{P}\right\} \subset\{1, \ldots, Q\}$ and $P \leq Q$, we will also use the notations $\mathcal{X}_{s-P a r}\left(f^{q_{1}}, \ldots, f^{q_{P}}\right), \mathcal{X}_{w-P a r}\left(f^{q_{1}}, \ldots, f^{q_{P}}\right)$ and $\mathcal{X}_{P a r}\left(f^{q_{1}}, \ldots, f^{q_{P}}\right)$ if (strict, weak) Pareto solutions for the criterion mapping $\left(f^{q_{1}}, \ldots, f^{q_{P}}\right)$ with range space in $\mathbb{R}^{P}$ are considered.

Since in this paper we are interested in structural results about the set of (strict, weka) Pareto solutions, we will assume throughout, that $\mathcal{X}_{P a r}$ is nonempty. Several conditions which guarantee $\mathcal{X}_{P a r} \neq \emptyset$ can e.g. be found in [11]. 
The general understanding is, that there is no ideal solution, i.e. there is no $x \in \mathcal{X}$ such that

$$
f^{q}(x)=\inf _{x \in \mathcal{X}} f^{q}(x) \forall q=1, \ldots, Q
$$

As a consequence of these two basic assumptions, $\mathcal{X}_{\text {Par }}$ contains at least two points.

Geometrically, the optimality definitions presented above can be characterized using level curves

$$
L_{=}^{q}(z):=\left\{x \in \mathcal{X}: f^{q}(x)=z\right\}
$$

level sets

$$
L_{\leq}^{q}(z):=\left\{x \in \mathcal{X}: f^{q}(x) \leq z\right\}
$$

and strict level sets

$$
L_{<}^{q}(z):=\left\{x \in \mathcal{X}: f^{q}(x)<z\right\} .
$$

A proof of the following Theorem can be found in [12].

Theorem 2.1 Let $x \in \mathcal{X}$ and $z_{q}:=f^{q}(x)$ for $q \in \mathcal{Q}$. Then the following hold:

1. $x$ is a strict Pareto solution if and only if

$$
\bigcap_{q \in \mathcal{Q}} L_{\leq}^{q}\left(z_{q}\right)=\{x\}
$$

2. $x$ is a Pareto solution if and only if

$$
\bigcap_{q \in \mathcal{Q}} L_{\leq}^{q}\left(z_{q}\right)=\bigcap_{q \in \mathcal{Q}} L_{=}^{q}\left(z_{q}\right)
$$

3. $x$ is a weak Pareto solution if and only if

$$
\bigcap_{q \in \mathcal{Q}}\left(L_{<}^{q}\left(z_{q}\right)\right)=\emptyset
$$

An immediate consequence of Theorem 2.1 is the following result, which states that if a point $x$ is a weak or a strict Pareto solution with respect to a subset of the objectives it is so with respect to all $Q$ criteria. 
Corollary 2.1 Let $x \in X$ and $\left\{q_{1}, \ldots, q_{P}\right\} \subseteq \mathcal{Q}$. Then the following hold:

$$
\begin{aligned}
& \text { 1. } x \in \mathcal{X}_{w-P a r}\left(f^{q_{1}}, \ldots, f^{q_{P}}\right) \Rightarrow x \in \mathcal{X}_{w-P a r}\left(f^{1}, \ldots, f^{Q}\right) . \\
& \text { 2. } x \in \mathcal{X}_{s-\text { Par }}\left(f^{q_{1}}, \ldots, f^{q_{P}}\right) \Rightarrow x \in \mathcal{X}_{s-\operatorname{Par}}\left(f^{1}, \ldots, f^{Q}\right) .
\end{aligned}
$$

Stronger results have been obtained by researchers, who have investigated the structure of $\mathcal{X}_{w-P a r}$. Let us assume from now on that $\mathcal{X}$ is a convex set and that $f^{q}, q=1, \ldots, Q$ are convex or strictly quasi-convex functions. A function $f^{q}$ is strictly quasi-convex, if for each $x$ and $y \in \mathbb{R}^{n}$

$$
f(\lambda x+(1-\lambda) y) \leq \max \{f(x), f(y)\}
$$

for all $\lambda \in(0,1)$ and strict inequality holds whenever $f(x) \neq f(y)$.

The representation

$$
\mathcal{X}_{w-P a r}=\bigcup_{\left\{q_{1}, \ldots, q_{P}\right\} \subset \mathcal{Q}: P \leq n+1} \mathcal{X}_{P a r}\left(f^{q_{1}}, \ldots, f^{q_{P}}\right)
$$

of $\mathcal{X}_{w-\text { Par }}$ in terms of Pareto solutions of subproblems of (MOP) has been proven in [7] for convex objectives. Later the same result has been obtained for upper semi-continuous strictly quasi-convex objectives in [8].

We will now proceed to show that equation (1) cannot be generalized to (even continuous) quasi-convex criteria, and present a weaker result, which is still true.

\section{Quasi-Convex Objectives}

A function $f$ is quasi-convex if for each $x$ and $y \in \mathbb{R}^{n}$

$$
f(\lambda x+(1-\lambda) y) \leq \max \{f(x), f(y)\}
$$


for all $\lambda \in(0,1)$. An appealing feature of quasi-convex functions is that they can be characterized in terms of level sets. The following Lemma is well known, see e.g. [13].

Lemma 3.1 $f: \mathbb{R}^{n} \rightarrow \mathbb{R}$ is quasi-convex if and only if $L_{\leq}(z)$ is convex for all $z \in \mathbb{R}$.

We show that (1) is not true for quasi-convex functions.

Example 3.1 Let $\mathcal{X}=\mathbb{R}$ and consider the three piecewise linear quasi-convex criteria shown in Figure 1.

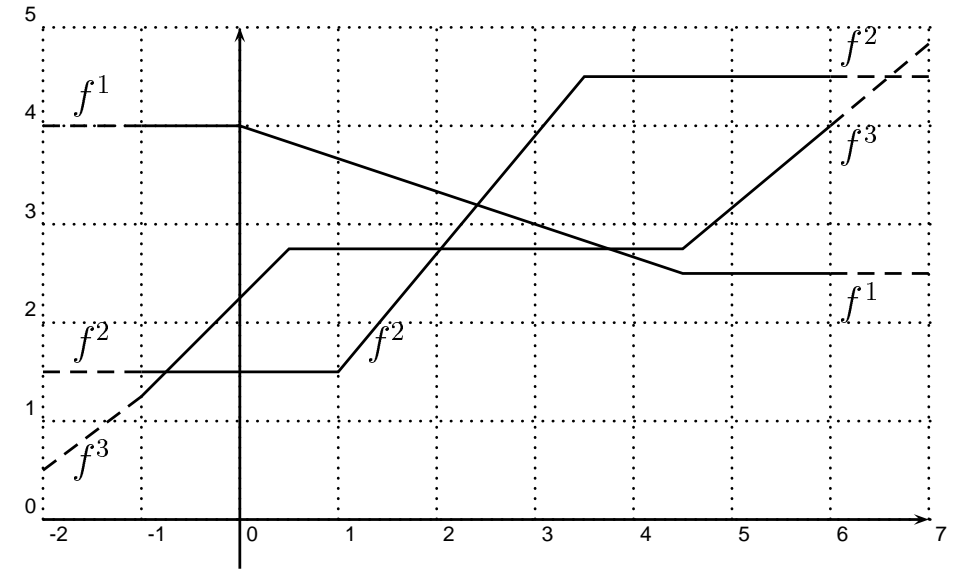

Figure 1: Three quasi-convex criteria

We observe the following:

$$
\begin{aligned}
\mathcal{X}_{\text {Par }}\left(f^{1}\right) & =[4.5, \infty) \\
\mathcal{X}_{\text {Par }}\left(f^{2}\right) & =(-\infty, 1] \\
\mathcal{X}_{\text {Par }}\left(f^{3}\right) & =\emptyset \\
\mathcal{X}_{\text {Par }}\left(f^{1}, f^{2}\right) & =[1,3.5) \cup[4.5, \infty) \\
\mathcal{X}_{\text {Par }}\left(f^{1}, f^{3}\right) & =(0,0.5) \cup\{4.5\} \\
\mathcal{X}_{\text {Par }}\left(f^{2}, f^{3}\right) & =\emptyset
\end{aligned}
$$


Therefore we have

$$
\bigcup_{q \in \mathcal{Q}} \mathcal{X}_{\text {Par }}\left(f^{q}\right) \cup \bigcup_{\left\{q_{1}, q_{2}\right\} \subset \mathcal{Q}} \mathcal{X}_{\text {Par }}\left(f^{q_{1}}, f^{q_{2}}\right)=\mathbb{R} \backslash[3.5,4.5) \neq \mathcal{X}_{w-P a r}=\mathbb{R}
$$

The Pareto set is $\mathcal{X}_{\text {Par }}=(0,0.5) \cup[1,3.5) \cup\{4.5\}$. Therefore not even the inclusion

$$
\mathcal{X}_{w-P a r} \subset \bigcup_{\left\{q_{1}, \ldots, q_{P}\right\} \subset \mathcal{Q}} \mathcal{X}_{P a r}\left(f^{q_{1}}, \ldots, f^{q_{P}}\right)
$$

is satisfied, without limitation of the number of objectives of subproblems. Note that due to Corollary 2.1 the reverse inclusion of (2) always holds. Thus, a representation of $\mathcal{X}_{w-P a r}$ in terms of Pareto solutions of subproblems is impossible.

However, we can obtain a representation similar to (1), if we replace $\mathcal{X}_{P a r}$ on the right hand side by $\mathcal{X}_{w-P a r}$. This result makes use of Helly's theorem on the intersection of convex sets stated below (see [14] and [15] for proofs).

Theorem 3.1 (Helly's Theorem) Let $C_{1}, \ldots, C_{Q}, Q \geq n+1$ be convex sets in $\mathbb{R}^{n}$. Then

$$
\bigcap_{q=1}^{Q} C_{q} \neq \emptyset \Leftrightarrow \forall q_{1}, \ldots, q_{n+1} \in \mathcal{Q}: C_{q_{1}} \cap \ldots \cap C_{q_{n+1}} \neq \emptyset,
$$

or equivalently

$$
\bigcap_{q=1}^{Q} C_{q}=\emptyset \Leftrightarrow \exists q_{1}, \ldots, q_{n+1} \in \mathcal{Q}: C_{q_{1}} \cap \ldots \cap C_{q_{n+1}}=\emptyset .
$$

We obtain the structure result for $\mathcal{X}_{w-P a r}$ in the quasi-convex case if we combine the results of Section 2 and Helly's Theorem. An immediate consequence of Theorem 2.1, Corollary 2.1, Lemma 3.1, and Theorem 3.1 is the following result.

Corollary 3.1 Suppose that $f^{1}, \ldots, f^{Q}$ are quasi-convex. Then a point $x \in \mathcal{X}$ is weakly Pareto optimal if and only if there exists a subset $\left\{f^{q_{1}}, \ldots, f^{q_{P}}\right\}$ of objective functions, $P \leq n+1$, such that $x \in \mathcal{X}_{w-P a r}\left(f^{q_{1}}, \ldots, f^{q_{P}}\right)$. 
In other words

$$
\mathcal{X}_{w-P a r}=\bigcup_{\left\{q_{1}, \ldots, q_{P}\right\} \subset \mathcal{Q}: P<n+1} \mathcal{X}_{w-P a r}\left(f^{q_{1}}, \ldots, f^{q_{P}}\right)
$$

Corollary 3.1 implies that, in order to obtain the weakly Pareto optimal points, it is sufficient to solve all subproblems with no more than $n+1$ criteria. When $n=2$ this means that in the worst case $O\left(Q^{3}\right)$ problems with up to three criteria have to be solved. The practical relevance for (MOP) with $Q \gg 2$, as is the case in location theory, is immediate.

\section{Strictly Quasi-Convex Objectives}

In the main part of the paper we consider strictly quasi-convex (MOP), i.e. $f^{q}: \mathbb{R}^{n} \rightarrow \mathbb{R}$ are strictly quasi-convex functions for all $q \in \mathcal{Q}$ and $\mathcal{X}$ is a convex subset of $\mathbb{R}^{n}$. Since strictly quasi-convex functions are quasi-convex, all level sets will be convex.

We have already mentioned the structure result for (upper semi-continuous) strictly quasiconvex functions from [8].

Lemma 4.1 Assume that $f^{1}, \ldots, f^{Q}$ are upper semi-continuous and strictly quasi-convex. Then

$$
\mathcal{X}_{w-P a r}=\bigcup_{\left\{q_{1}, \ldots, q_{P}\right\}: P \leq n+1} \mathcal{X}_{P a r}\left(f^{q_{1}}, \ldots, f^{q_{P}}\right)
$$

The question is, whether a similar result can be proved for $\mathcal{X}_{\text {Par }}$. I.e. is it possible to distinguish weakly Pareto optimal points from Pareto optimal points using at most $n+1$ of the criteria? We cannot answer this question in general, but we have an affirmative result for the case of problems in two variables.

We will show that, for any point $x \in \mathcal{X}$ it is possible to decide if it is Pareto optimal or not using at most 3 of the criteria at a time, i.e. by solving at most $O\left(Q^{3}\right)$ subproblems, 
which is the same as for determination of $\mathcal{X}_{w-\text { Par }}$.

Before we can prove the main result of the paper (Theorem 4.3) in several steps, we have to introduce some notation and prove some preliminary results. We will use the notion of the dimension of a convex set $\mathcal{X}$ which is defined as the dimension of the affine subspace spanned by $\mathcal{X}$, i.e.

$$
\operatorname{dim}(\mathcal{X}):=\operatorname{dim}(\operatorname{span}(\mathcal{X}))
$$

Furthermore we will refer to the interior, relative interior and relative boundary of $\mathcal{X}$ as $\operatorname{int} \mathcal{X}, \operatorname{ri} \mathcal{X}$, and $\operatorname{rbd} \mathcal{X}$, respectively.

First a sufficient condition for Pareto optimality of a feasible point $x$ is given. Note that Lemma 4.2 does not rely on any convexity assumption.

Lemma 4.2 Let $x \in \mathcal{X}$ and suppose that there are $\mathcal{J}_{1}, \ldots, \mathcal{J}_{k} \subset \mathcal{Q},\left|\mathcal{J}_{i}\right|<Q$ such that $\cup_{i=1}^{k} \mathcal{J}_{i}=\mathcal{Q}$ and that $x \in \mathcal{X}_{\text {Par }}\left(f^{q}: q \in \mathcal{J}_{i}\right)$ for all $i=1, \ldots, k$. Then $x \in \mathcal{X}_{\text {Par }}$

\section{Proof.}

Since $x \in \mathcal{X}_{\text {Par }}\left(f^{q}: q \in \mathcal{J}_{i}\right)$ for all $i$, we have

$$
\begin{aligned}
\bigcap_{q \in \mathcal{J}_{i}} L_{\leq}\left(f^{q}(x)\right) & =\bigcap_{q \in \mathcal{J}_{i}} L_{=}\left(f^{q}(x)\right) \quad i=1, \ldots, k \\
\Rightarrow \bigcap_{i=1}^{k} \bigcap_{q \in \mathcal{J}_{i}} L_{\leq}\left(f^{q}(x)\right) & =\bigcap_{i=1}^{k} \bigcap_{q \in \mathcal{J}_{i}} L_{=}\left(f^{q}(x)\right) .
\end{aligned}
$$

and from the assumption on the $\mathcal{J}_{i}$ :

$$
\bigcap_{q=1}^{Q} L_{\leq}\left(f^{q}(x)\right)=\bigcap_{q=1}^{Q} L_{=}\left(f^{q}(x)\right) .
$$

Observe that the claim of Lemma 4.2 holds trivially as a necessary and sufficient condition, if we choose $\mathcal{J}=\mathcal{Q}$., but note that the condition is not necessary in general otherwise: 
The point $x=2.5 \in \mathcal{X}_{\text {Par }}$ in Example 3.1 is Pareto optimal for the subproblem with $f^{1}$ and $f^{2}$, but no other subset.

To prove that the condition is necessary for strictly quasi-convex objectives (at least when $\mathcal{X} \subset \mathbb{R}^{2}$ ), we proceed with some technical Lemmas.

Lemma 4.3 Let $f$ be a strictly quasi-convex function. Then for all $z>\inf _{x \in \mathcal{X}} f(x)$ it holds that $L_{=}(z) \subset \operatorname{rbd} L_{\leq}(z)$.

\section{Proof.}

We show that $L_{=}(z) \cap \operatorname{ri} L_{\leq}(z) \neq \emptyset$ implies $z=\inf _{x \in \mathcal{X}} f(x)$.

Assume that $x \in L_{=}(z) \cap \operatorname{ri} L_{\leq}(z) \neq \emptyset$ and choose $x^{1} \in L_{\leq}(z), x^{1} \neq x$. Then there exist $x^{2} \in L_{\leq}(z)$ and $0<\alpha<1$ such that $x=\alpha x^{1}+(1-\alpha) x^{2}$. Suppose that $f\left(x^{1}\right) \neq f\left(x^{2}\right)$. Then by strict quasi-convexity and because $x^{1}, x^{2} \in L_{\leq}(z)$

$$
z=f(x)<\max \left\{f\left(x^{1}\right), f\left(x^{2}\right)\right\} \leq z,
$$

a contradiction. Therefore $f\left(x^{1}\right)=f\left(x^{2}\right)$. Since $x^{1} \in L_{\leq}(z) \backslash\{x\}$ was arbitrary, we get

$$
z=f(x) \leq \max \left\{f\left(x^{1}\right), f\left(x^{2}\right)\right\}=f\left(x^{1}\right)=z
$$

and $f\left(x^{1}\right)=f(x)$ for all $x^{1} \in L_{\leq}(z)$. Therefore $L_{\leq}(z)=L_{=}(z)$ which implies $z=$ $\min _{x \in \mathcal{X}} f(x)$

From now on we restrict ourselves to the case that $\mathcal{X} \subset \mathbb{R}^{2}$.

Lemma 4.4 Let $\mathcal{X} \subset \mathbb{R}^{2}$ such that $\operatorname{dim} \mathcal{X}=2$ and let $f$ be a strictly quasi-convex upper semi-continuous function. Then $\operatorname{dim} L_{\leq}\left(f\left(x^{*}\right)\right) \leq 1$ implies that $f\left(x^{*}\right)=\inf _{x \in \mathcal{X}} f(x)$.

\section{Proof.}


The case of $\operatorname{dim} L_{\leq}\left(f\left(x^{*}\right)\right)=0$ is trivial. So assume that $\operatorname{dim} L_{\leq}\left(f\left(x^{*}\right)\right)=1$ and suppose there is an $x \in \mathcal{X}$ such that $f(x)<f\left(x^{*}\right)$. Then let $U:=U_{\epsilon}(x) \cap \mathcal{X}$. By the assumptions, $U \backslash L_{\leq}\left(f\left(x^{*}\right)\right)$ is two-dimensional. Therefore we have $f\left(x^{\prime}\right)>f\left(x^{*}\right)$ for all $x^{\prime}$ in that set. If we choose $x_{\lambda}:=\lambda x^{\prime}+(1-\lambda) x$ we have

$$
f\left(x_{\lambda}\right)>f\left(x^{*}\right) \quad \forall \lambda \in(0,1]
$$

However, if we let $\lambda$ take arbitrarily small positive values, the upper semi-continuity implies $f(x) \geq f\left(x^{*}\right)$, contradicting our assumption.

The next Theorem provides the converse of Lemma 4.2 for Pareto optimal points, which are not strictly Pareto optimal.

Theorem 4.1 Suppose that $x^{*} \in \mathcal{X}_{\text {Par. }}$. Then either $x^{*}$ is strictly Pareto optimal or there exist subsets $\mathcal{J}_{1}, \ldots, \mathcal{J}_{k} \subset \mathcal{Q},\left|\mathcal{J}_{i}\right| \leq 3$ such that $\cup_{i=1}^{k} \mathcal{J}_{i}=\mathcal{Q}$ and that $x \in \mathcal{X}_{\text {Par }}\left(f^{q}: q \in \mathcal{J}_{i}\right)$ for all $i=1, \ldots, k$.

\section{Proof.}

Suppose $x^{*}$ is not strictly Pareto optimal. Then there is no $q \in \mathcal{Q}$ such that $x^{*}$ is a unique minimizer of $f^{q}$ over $X$. We define

$$
S:=\bigcap_{q=1}^{Q} L_{\leq}\left(f^{q}\left(x^{*}\right)\right) .
$$

First we show that $\operatorname{dim} S=1$.

1. Since $x^{*} \notin \mathcal{X}_{s-\text { Par }}$ we know that $|S| \geq 2$. Because $S$ is convex this implies $\operatorname{dim} S \geq 1$.

2. Assume that $\operatorname{dim} S=2$. Then $\operatorname{int} S \neq \emptyset$. Hence we can choose $\hat{x} \in \operatorname{int} S$. From Theorem 2.1 we know that $S:=\bigcap_{q=1}^{Q} L_{=}\left(f^{q}\left(x^{*}\right)\right)$ and therefore $S \subseteq L_{=}\left(f^{q}\left(x^{*}\right)\right)$ for all $q=1, \ldots, Q$. Thus, $\hat{x} \in \operatorname{int} L_{=}\left(f^{q}\left(x^{*}\right)\right)$. From the proof of Lemma 4.3 we conclude that $f^{q}\left(x^{*}\right)=\inf _{x \in \mathcal{X}} f^{q}(x)$ for all $q=1, \ldots, Q$, which implies that $x^{*}$ is an ideal solution. This contradiction implies $\operatorname{dim} S \leq 1$. 
Next we show that, without loss of generality, we can assume that for all $q$ there is a line separating $S$ and $L_{\leq}\left(f^{q}\left(x^{*}\right)\right)$, i.e. we show

$$
\operatorname{int} L_{\leq}\left(f^{q}\left(x^{*}\right)\right) \cap \operatorname{ri} S=\emptyset .
$$

Suppose, to the contrary, that $\operatorname{int} L_{\leq}\left(f^{q}\left(x^{*}\right)\right) \cap \operatorname{ri}(S) \neq \emptyset$. Note that this is only possible if $\operatorname{dim} L_{\leq}\left(f^{q}\left(x^{*}\right)\right)=2$. So let $\hat{x} \in \operatorname{int} L_{\leq}\left(f^{q}\left(x^{*}\right)\right) \cap \operatorname{ri} S$. From $\hat{x} \in S$ we have (as above) $\hat{x} \in L_{=}\left(f^{q}\left(x^{*}\right)\right)$ and from $\hat{x} \in \operatorname{int} L_{\leq}\left(f^{q}\left(x^{*}\right)\right)$ by Lemma 4.3 we get $f^{q}(\hat{x})=\inf _{x \in \mathcal{X}} f^{q}(x)$. Therefore $\hat{x}$ (and $x^{*}$ ) are minimizers of $f^{q}$. Therefore $x^{*}$ is Pareto optimal for $f^{q}$. If we prove that $x^{*}$ is also Pareto optimal for the remaining objectives, we can choose $\mathcal{J}_{1}=\{q\}$ and restrict ourselves to $\mathcal{Q} \backslash\{q\}$.

To do so, consider $S^{\prime}:=\bigcap_{i \in \mathcal{Q} \backslash\{q\}} L_{\leq}\left(f^{i}\left(x^{*}\right)\right)$. We have that $S \subset S^{\prime}$ and that $\operatorname{dim} S^{\prime}=1$ (from convexity). We will show that $S^{\prime}:=\bigcap_{i \in \mathcal{Q} \backslash\{q\}} L_{=}\left(f^{i}\left(x^{*}\right)\right)$. If $S=S^{\prime}$ there is nothing to prove, so assume there is an $x \in S^{\prime} \backslash S$. Suppose that there is an $x^{\prime} \in S^{\prime}$ and a $q^{\prime}$ s.t. $f^{q^{\prime}}\left(x^{\prime}\right)<f^{q^{\prime}}(x)$, i.e. $x^{\prime} \in L_{<}\left(f^{q^{\prime}}\left(x^{*}\right)\right)$. Obviously, $x^{\prime} \in S^{\prime} \backslash S$. Now take $\hat{x} \in \operatorname{ri} S$ and consider $x_{\lambda}:=\lambda x^{\prime}+(1-\lambda) \hat{x}$. We obtain

$$
f^{q^{\prime}}\left(x_{\lambda}\right) \leq \max \left\{f^{q^{\prime}}\left(x^{\prime}\right), f^{q^{\prime}}(\hat{x})\right\}<f^{q^{\prime}}(\hat{x})
$$

(because $\left.f^{q^{\prime}}\left(x^{\prime}\right)<f^{q^{\prime}}(x) \leq f^{q^{\prime}}\left(x^{*}\right)=f^{q^{\prime}}(\hat{x})\right)$, which is true for all $\lambda \in(0,1]$. But there must exist some $\lambda^{*}<1$ such that $x_{\lambda^{*}} \in \operatorname{rbd} S$, which implies the existence of an $x \in S$ with $f^{q^{\prime}}(x)<f^{q^{\prime}}\left(x^{*}\right)$, an impossibility. By the contradiction we know that $x^{*}$ is Pareto optimal for the remaining objectives.

Now, we can assume w.l.o.g that $\operatorname{int} L_{\leq}\left(f^{q}\left(x^{*}\right)\right) \cap \operatorname{ri} S=\emptyset$. Therefore, for each $q$ there exists a line

$$
h_{q}:=\left\{x \in \mathbb{R}^{2}:<a_{q}, x>-b_{q}=0\right\}
$$

such that

$$
<a_{q}, x>\leq<a_{q}, y>\quad \forall y \in S \forall x \in L_{\leq}\left(f^{q}\left(x^{*}\right)\right)
$$


But since $S \subset L_{\leq}\left(f^{q}\left(x^{*}\right)\right)$ we know that $S \subset h_{q}$ for each $q$. Because $\operatorname{dim} S=1$ we see that all these lines are one and the same, i.e. $h_{q}=h \forall q=1, \ldots, Q$. Denote by $h^{+}$and $h^{-}$the open positive and negative half-plane w.r.t. $h$.

We show that not all sets $L_{\leq}\left(f^{q}\left(x^{*}\right)\right)$ can have nonempty intersection with the same open halfspace (let's say $\left.h^{+}\right)$. This can only be true if all $L_{\leq}\left(f^{q}\left(x^{*}\right)\right)$ have dimension two. Suppose this is the case. All these sets have the one-dimensional set $S$, which is contained in their boundary, in common. Then convexity and the fact that they are all on one side of $h($ and $S$ ) implies that their interiors have nonempty intersection. But due to Lemma $4.3 \operatorname{int} L_{\leq}\left(f^{q}\left(x^{*}\right)\right) \subset L_{<}\left(f^{q}\left(x^{*}\right)\right)$, and such points contradict Pareto optimality of $x^{*}$.

Now we can choose subsets $\left|\mathcal{J}_{i}\right|$ of at most three level curves $L_{\leq}\left(f^{q}\left(x^{*}\right)\right)$ such that their intersection is contained in $h$. With similar arguments used to prove the w.l.o.g. part above it follows that

$$
\bigcap_{q \in \mathcal{J}_{i}} L_{\leq}\left(f^{q}\left(x^{*}\right)\right)=\bigcap_{q \in \mathcal{J}_{i}} L_{=}\left(f^{q}\left(x^{*}\right)\right)
$$

and $x^{*} \in \mathcal{X}_{\text {Par }}\left(f^{q}: q \in \mathcal{J}_{i}\right)$.

Note that in general the sets $\mathcal{J}_{i}$ are not disjoint. To conclude our results, we still have to show that we can also determine Pareto optimality of the strictly Pareto optimal solutions by looking at at most 3 objectives at a time.

Theorem 4.2 Let $x^{*}$ be a strictly Pareto optimal solution. Then either $x^{*}$ is strictly Pareto optimal for a subset of at most 3 objectives, or there exist subsets $\mathcal{J}_{1}, \ldots, \mathcal{J}_{k} \subset \mathcal{Q},\left|\mathcal{J}_{i}\right| \leq 3$ such that $\cup_{i=1}^{k} \mathcal{J}_{i}=\mathcal{Q}$ and that $x \in \mathcal{X}_{\text {Par }}\left(f^{q}: q \in \mathcal{J}_{i}\right)$ for all $i=1, \ldots, k$.

\section{Proof.}

Suppose that there is no subset $\mathcal{J} \subset \mathcal{Q},|\mathcal{J}| \leq 3$ such that $x^{*} \in \mathcal{X}_{s-\operatorname{Par}}\left(f^{q}: q \in \mathcal{J}\right)$. Since $x^{*}$ is strictly Pareto optimal, it is also weakly Pareto optimal. From Lemma 4.1 we 
know that there is a set $\mathcal{J}$ of at most three objectives, such that $x^{*} \in \mathcal{X}_{\text {Par }}\left(f^{q}: q \in \mathcal{J}\right)$. According to our assumption, $x^{*}$ is not strictly Pareto optimal for these objectives.

Considering the objectives $f^{q}, q \in \mathcal{Q} \backslash \mathcal{J}$ we can distinguish the following cases.

1. $x^{*}$ is strictly Pareto optimal for the remaining objectives. Then we can just consider $\mathcal{Q} \backslash \mathcal{J}$ instead of $J$.

2. $x^{*}$ is weakly Pareto optimal for the remaining objectives. Then we can apply Lemma 4.1 again, to get another subset of criteria and consider the remaining ones.

3. $x^{*}$ is Pareto optimal, but not strictly Pareto optimal for the remaining objectives. Then we can apply Theorem 4.1 to the problem with objectives $f^{q}, q \in \mathcal{Q} \backslash \mathcal{J}$ and are done.

4. $x^{*}$ is not even weakly Pareto optimal. Considerations as in the proof of Theorem 4.1 show that this can only happen if

$$
S:=\bigcap_{q \in \mathcal{Q} \backslash \mathcal{J}} L_{\leq}\left(f^{q}\left(x^{*}\right)\right)
$$

has dimension two. But $\mathcal{X}_{\text {Par }}\left(f^{q}: q \in \mathcal{J}\right)$ defines a line (recall that $x^{*}$ is not strictly Pareto optimal for objectives in $\mathcal{J}$ ). Analogous to the proof of Theorem 4.1 we can show that $S$ lies in exactly one of the (closed) halfspaces defined by this line (or, eventually, the normal of that line). Then we can again choose the appropriate subsets as described in the proof of Theorem 4.1 .

Summarizing the previous results (Lemma 4.2, Theorems 4.1 and 4.2), we can state the main result of this paper.

Theorem 4.3 In a multicriteria optimization problem with upper semi-continuous strictly quasi-convex objectives and convex feasible set $\mathcal{X} \subset \mathbb{R}^{2}$, a feasible point $x^{*}$ is Pareto optimal 
if and only if there is a subset $\mathcal{J} \subset \mathcal{Q},|\mathcal{J}| \leq 3$ such that

$$
x^{*} \in \mathcal{X}_{s-P a r}\left(f^{q}: q \in \mathcal{J}\right)
$$

or there exist subsets $\mathcal{J}_{1}, \ldots, \mathcal{J}_{k} \subset \mathcal{Q},\left|\mathcal{J}_{i}\right| \leq 3$ such that $\cup_{i=1}^{k} \mathcal{J}_{i}=\mathcal{Q}$ and that $x \in$ $\mathcal{X}_{\text {Par }}\left(f^{q}: q \in \mathcal{J}_{i}\right)$ for all $i=1, \ldots, k$.

We remark that the proofs of Theorems 4.1 and 4.2 use the fact that $\mathcal{X} \subset \mathbb{R}^{2}$, when we show that the same hyperplane separates all $L_{\leq}\left(f^{q}\left(x^{*}\right)\right)$ from $S$. This cannot be generalized to higher dimensions. Therefore the question, if Theorem 4.3 is still valid for higher dimensional $\mathcal{X}$ is still open. We also note that Example 3.1 presented in Section 3 shows that we cannot generalize the result to quasi-convex objectives, not even if $\mathcal{X} \subset \mathbb{R}$, and the objectives are continuous.

Theorem 4.3 yields a prototype algorithm to solve a strictly quasi-convex (MOP) with two variables. We denote the set of all subsets of $\mathcal{Q}$ with no more than 3 elements by $\mathcal{Q}(3)$,

$$
\mathcal{Q}(3):=\{\mathcal{J} \subset \mathcal{Q}:|\mathcal{J}| \leq 3\} .
$$

For $q \in \mathcal{J}$ we use the following abbreviations:

$$
\begin{aligned}
& L_{\leq}^{q}:=L_{\leq}^{q}\left(f^{q}(x)\right) \\
& L_{\leq}:=\bigcap_{q \in \mathcal{J}} L_{\leq}^{q} .
\end{aligned}
$$

Note that by the definition of level sets $x \in L_{\leq}$and therefore $L_{\leq} \neq \emptyset$.

\section{Algorithm for strictly quasi-convex (MOP) with 2 variables}

1. Let $k:=0$, choose $\mathcal{J} \in \mathcal{Q}(3)$.

2. Let $\mathcal{Q}(3):=\mathcal{Q}(3) \backslash \mathcal{J}$.

3. If $\operatorname{dim} L_{\leq}=0 \mathrm{STOP}, x^{*}$ is strictly Pareto optimal for (MOP). 
4. If $x^{*}$ is Pareto optimal for $\left\{f^{q}: q \in \mathcal{J}\right\}$ let $k:=k+1, \mathcal{J}_{k}:=\mathcal{J}$

- If $\bigcup_{i=1}^{k} \mathcal{J}_{k}=\mathcal{Q}$ STOP, $x^{*}$ is Pareto optimal for (MOP).

5. If $\mathcal{Q}(3)$ is not empty

- If $|\mathcal{J}|<3$ choose $q \in \mathcal{Q} \backslash \mathcal{J}$, let $\mathcal{J}=\mathcal{J} \cup\{q\}$ and go to 2 .

- Otherwise choose a new $\mathcal{J}$ from $\mathcal{Q}(3)$ and go to 2 .

6. STOP, $x^{*}$ is not Pareto optimal for (MOP).

The algorithm will terminate after checking $x^{*}$ for Pareto optimality for at most all of the $O\left(Q^{3}\right)$ subproblems with no more than three objective functions. Assuming that checking intersections of two or three convex sets can be done efficiently, we have an efficient algorithm for checking Pareto optimality. It is important to note that, for special types of objective functions the above result indeed leads to efficient algorithms for solving (i.e. determining all Pareto solutions of) multicriteria optimization problems. This is the case in location theory, where we refer to [16], [10], and [17] for details.

Lemma 4.1 and Theorem 4.3 show how to decide (weak) Pareto optimality of a point $x^{*}$ by considering subproblems with at most 3 objective functions (essentially, checking intersections of 3 convex level sets). To conclude this section we briefly address the case of strict Pareto optimality. Note that the criterion in Theorem 4.2 does not necessarily imply that we can decide strict Pareto optimality of $x^{*}$, only its Pareto optimality is shown.

However, the geometrical characterization of strict Pareto optimality provided by Theorem 2.1 seems to be easier than that of Pareto optimality. But the following example shows that a lower bound on the number of criteria needed to decide strict Pareto optimality is $2 n$.

In Figure 2, $x$ belongs to both $\mathcal{X}_{\text {Par }}\left(f^{1}, f^{3}\right)$ and $\mathcal{X}_{\text {Par }}\left(f^{2}, f^{4}\right)$ as well as $\mathcal{X}_{s-\text { Par }}\left(f^{1}, \ldots, f^{4}\right)$ but is not Pareto optimal for any set of one or three objectives. The Figure also illustrates 


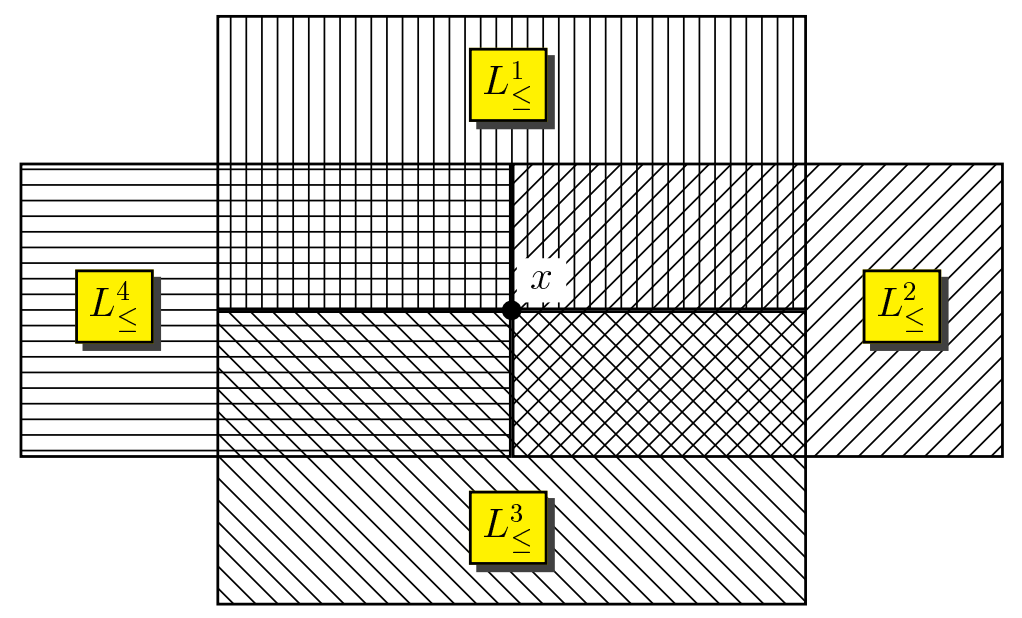

Figure 2: Intersection of 4 level sets

the third case in the proof of Theorem 4.2.

\section{An Illustrative Example}

We consider an Example with four objectives and $\mathcal{X}=[-5,5] \times[-5,5] \subseteq \mathbb{R}^{2}$. The objectives are

$$
\begin{aligned}
& f^{1}(x, y)=(x-2.5)^{2}+(y-0.5)^{2}-6.5 \\
& f^{2}(x, y)=2 x^{2}+\frac{1}{2}(y-3)^{2}-4.5 \\
& f^{3}(x, y)=y-5 \\
& f^{4}(x, y)=2 x+3
\end{aligned}
$$

First of all we show that none of the objectives is nonessential. In the following table we list the 4 objectives $f^{i}$ in the first column. The second and third columns show four points $x^{i}$ which are Pareto solutions for the (MOP)

$$
\min _{x \in \mathcal{X}}\left(f^{1}, f^{2}, f^{3}, f^{4}\right)
$$


and their respective objective values. The fourth and fifth column show points $x_{d}^{i}$ which dominate $x^{i}$ in the (MOP)

$$
\min _{x \in \mathcal{X}} \hat{f}_{i}
$$

where $\hat{f}_{i}$ denotes the criterion mapping $f$ with $f^{i}$ dropped.

\begin{tabular}{|c|c|c|c|c|c|c|c|c|c|c|c|c|}
\hline$f^{i}$ & \multicolumn{2}{|c|}{$x^{i}$} & \multicolumn{4}{|c|}{$f\left(x^{i}\right)$} & \multicolumn{2}{|c|}{$x_{d}^{i}$} & \multicolumn{4}{|c|}{$\hat{f}_{i}\left(x_{d}^{i}\right)$} \\
\hline$f^{1}$ & $(2.5$ & $0.5)$ & $(-6.5$, & 11.25, & -4.5 & 8) & $(0$, & 0) & $(\bullet$ & 0 & -5 , & 3) \\
\hline$f^{2}$ & $(0$ & 3) & $(6$, & -4.5 & -2 & 3) & $(0$, & 1) & $(0$, & $\bullet$ & -4 , & 3) \\
\hline$f^{3}$ & $(1$, & $-5)$ & $(26$, & 29.5 & -10 & 5) & $(0$, & $-2)$ & $(6$, & 8, & $\bullet$, & 3) \\
\hline$f^{4}$ & $(-5$ & 1) & $(50$ & 47.5 & -4 & $-7)$ & $(-4$, & 0) & $(36$, & 32 , & -5 , & •) \\
\hline
\end{tabular}

Note that $x^{1}$ and $x^{2}$ are Pareto optimal because they are the (unique) minimizers of $f^{1}$ and $f^{2}$, respectively. Points $x^{3}$ and $x^{4}$ are minimizers of $f^{3}$ and $f^{4}$ over $\mathcal{X}$, respectively. Furthermore they are Pareto optimal for

$$
\min \left\{\hat{f}_{3}(x, y):(x, y) \in \mathcal{X}, y=-5\right\}
$$

the Pareto set of which is $[-5,2.5] \times\{-5\}$ and for

$$
\min \left\{\hat{f}_{4}(x, y):(x, y) \in \mathcal{X}, x=-5\right\}
$$

with the Pareto set $\{-5\} \times[-5,3]$, respectively. Both facts imply that $x^{3}$ and $x^{4}$ are Pareto optimal for the original (MOP).

Now we apply the prototype procedure described after Theorem 4.3 for two feasible points.

First, we consider $x=(0,0)$. The corresponding level curves are shown in Figure 3. From $\bigcap_{q=1}^{4} L_{\leq}^{q}(x)=\{x\}$ with Theorem 2.1 we see that $x$ is a strict Pareto solution.

We choose $\mathcal{J}=\{1,4\}$. As

$$
\operatorname{dim} \bigcap_{q \in \mathcal{J}} L_{\leq}^{q}=2
$$




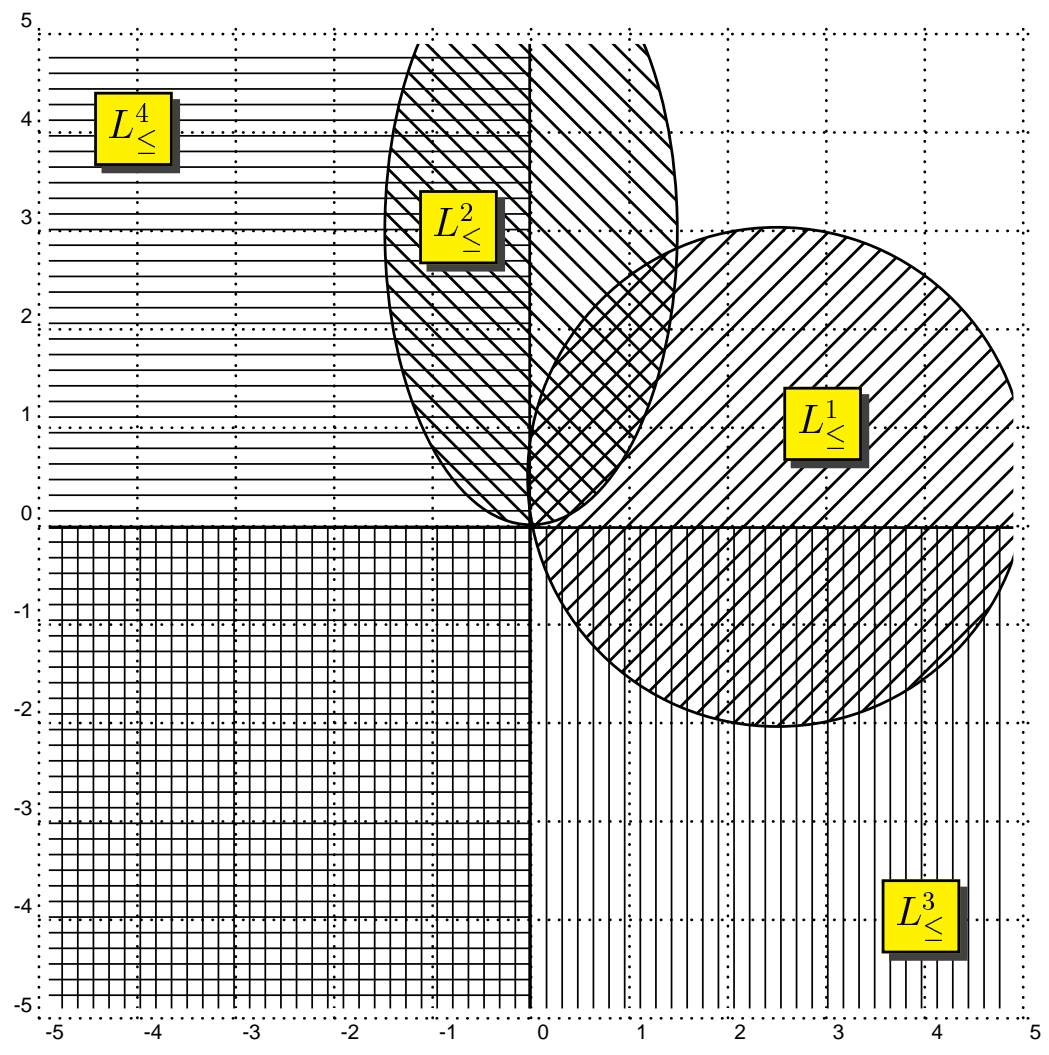

Figure 3: Level sets for $x=(0,0)$

(the three points $(0,0),(0,1)$ and $(2.5-\sqrt{6.5}, 0.5)$ belong to both level sets) $x$ is not Pareto optimal for the objectives in $\mathcal{J}$. We choose in addition $q=2$. Now $\operatorname{dim}\left(L_{\leq} \cap L_{\leq}^{2}\right)=2$ and $x$ is not Pareto optimal for $\left(f^{1}, f^{2}, f^{4}\right)$ and we select a new $\mathcal{J}$.

We choose $\mathcal{J}=\{1,3\}$ and again

$$
\operatorname{dim} \bigcap_{q \in \mathcal{J}} L_{\leq}^{q}=2 .
$$

Now we choose $q=2$ to be added to $\mathcal{J}$. Then $\operatorname{dim}\left(L_{\leq} \cap L_{\leq}^{2}\right)=0$ implying that $x$ is strictly Pareto optimal.

Also note that if $\mathcal{J}=\{2,3\}$ is chosen initially, strict Pareto optimality is immediate, i.e. our results do not provide any information on which objectives really determine Pareto optimality of $x$. 
For a second point, let us try $x=(5,5)$. The corresponding level sets are depicted in Figure 4. Note that $L_{\leq}^{3}=L_{\leq}^{4}=\mathcal{X}$ in this case.

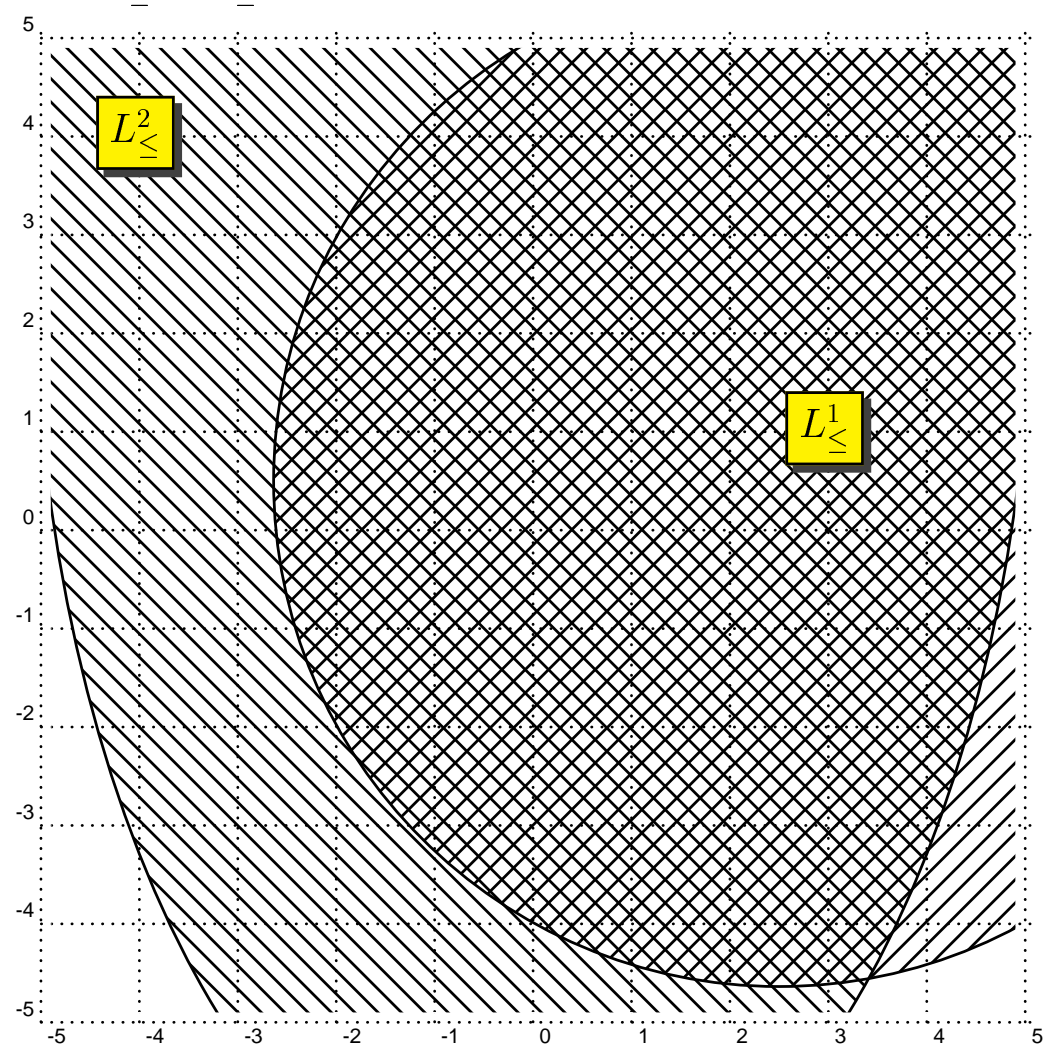

Figure 4: Level sets for $x=(5,5)$

Evidently, whatever the choice of $\mathcal{J}, \bigcap_{q \in \mathcal{J}} L_{\leq}^{q}$ will have dimension two, and the procedure will (correctly) stop with the conclusion that $x$ is not Pareto optimal. The geometric characterization of Theorem 2.1 is not satisfied for $x$, confirming this conclusion.

\section{Conclusions and Future Research}

In this paper we have discussed methods to decide Pareto optimality for a point in the decision space of (MOP) using only subsets of the set of criteria. This result extends previous work of [7] and [8] that characterizes weak Pareto solutions in terms of Pareto solutions of subproblems with at most $n+1$ criteria. 
First we have shown that this result cannot be generalized for quasi-convex objectives and we have given a weaker result in this case. For the case of strictly quasi-convex objectives we have extended the result of [8] to Pareto optimality, at least for problems with two variables, which are of fundamental importance in location theory. Our results yield a prototype algorithm for such problems. The question whether these results are true in higher dimensional problems remains open for now. We remark that a counterexample would be an (MOP) with at least three variables and at least five objectives.

We have also shown that similar results for strict Pareto optimality cannot be obtained.

Let us also mention that in the case $n=1$ Theorem 4.3 is still true of course, but not

helpful. It is well known that $\mathcal{X}_{P a r}$ and $\mathcal{X}_{w-\text { Par }}$ are connected, see [18]. Then these sets are intervals and their determination is equivalent to the solution of $Q$ single criterion convex minimization problems in $\mathbb{R}$.

As points for future research, let us mention that besides applications of the results presented here, which have already already been started in location theory (see [16], [10], and [17]), we will will be concerned with more general settings.

We also note that some question related to the topic of this paper are still open. For example it is not known if

$$
\mathcal{X}_{\text {Par }} \subset \bigcup_{\mathcal{J} \subset \mathcal{Q}:|\mathcal{J}| \leq n+1} \mathcal{X}_{\text {Par }}\left(f^{q}: q \in \mathcal{J}\right)
$$

holds for quasi-convex objectives.

\section{References}

[1] T. Gal. A note on size reduction of the objective functions matrix in vector maximum problems. In G. Fandel and T. Gal, editors, Multiple Criteria Decision Making 
Theory and Application, volume 177 of Lecture Notes in Economics and Mathematicsl Systems. Springer Verlag, Berlin, 1980.

[2] H. Leberling. On the Theory of Linear Vectormaximum Problems (in German). PhD thesis, RWTH Aachen, 1977.

[3] T. Gal and H. Leberling. Redundant objective functions in linera vector maximum problems and their determination. European Journal of Operational Research, 1:176$184,1977$.

[4] T. Gal and T. Hanne. Consequences of dropping nonessential objectives for the application of MCDM-methods. Paper presented at the EURO XV/INFORMS XXIV meeting, Barcelona, July 1997.

[5] C. Carlsson and R. Fuller. Multiple criteria decision making: The case for interdependence. Computers and Operations Research, 22:251-260, 1995.

[6] C. Carlsson and R. Fuller. Interdependence in fuzzy multiple objective programming. Fuzzy Sets and Systems, 65:19-29, 1994.

[7] J. Ward. Structure of efficient sets for convex objectives. Mathematics of Operations Research, 14(2):249-257, 1989.

[8] C. Malivert and N. Boissard. Structure of efficient sets for strictly quasi convex objectives. Journal of Convex Analysis, 1(2):143-150, 1994.

[9] J. Current, H. Min, and D. Schilling. Multiobjective analysis of facility location decisions. European Journal of Operational Research, 49:295-307, 1990.

[10] H.W. Hamacher and S. Nickel. Multicriteria planar location problems. European Journal of Operational Research, 94:66-86, 1996.

[11] Y. Sawaragi, H. Nakayama, and T. Tanino. Theory of Multiobjective Optimization. Academic Press, Orlando, 1985. 
[12] M. Ehrgott, H.W. Hamacher, K. Klamroth, S. Nickel, A. Schöbel, and M.M. Wiecek. A note on the equivalence of balance points and Pareto solutions in multiple-objective programming. Journal of Optimization Theory and Applications, 92(1):209-212, 1997.

[13] M.S. Bazaara, H.D. Sherali, and C.M. Shetty. Nonlinear Programming - Theory and Algorithms. John Wiley \& Sons, New York, 1993.

[14] F.A. Valentine. Convex Sets. McGraw-Hill, New York, 1964.

[15] E. Helly. On sets of convex bodies with common points (in German). Jahrbuch der Deutschen Mathematiker Vereinigung, 32:175-176, 1923.

[16] S. Nickel. Discretization of Planar Location Problems. Shaker Verlag, Aachen, 1995.

[17] S. Nickel. Bicriteria and restricted 2-facility Weber problems. Mathematical Methods of Operations Research, 45(2):167-195, 1997.

[18] A.R. Warburton. Quasiconcave vector maximization: Connectedness of the sets of Pareto-optimal and weak Pareto-optimal alternatives. Journal of Optimization Theory and Applications, 40:537-557, 1983. 